\title{
Geometric correction of APEX hyperspectral data
}

\section{Abstract}

Hyperspectral imagery originating from airborne sensors is nowadays widely used for the detailed characterization of land surface. The correct mapping of the pixel positions to ground locations largely contributes to the success of the applications. Accurate geometric correction, also referred to as "orthorectification", is thus an important prerequisite which must be performed prior to using airborne imagery for evaluations like change detection, or mapping or overlaying the imagery with existing data sets or maps. A so-called "ortho-image" provides an accurate representation of the earth's surface, having been adjusted for lens distortions, camera tilt and topographic relief. In this paper, we describe the different steps in the geometric correction process of APEX hyperspectral data, as applied in the Central Data Processing Center (CDPC) at the Flemish Institute for Technological Research (VITO, Mol, Belgium). APEX ortho-images are generated through direct georeferencing of the raw images, thereby making use of sensor interior and exterior orientation data, boresight calibration data and elevation data. They can be referenced to any userspecified output projection system and can be resampled to any output pixel size.

Keywords

Hyperspectral • APEX • Geometric correction • Airborne • Georeferencing - Boresight calibration

(C) University of Warsaw - Faculty of Geography and Regional Studies

Introduction

In 2004, at the time the Central Data Processing Center was being designed and developed, VITO decided to implement its own orthorectification $\mathrm{C}++$ module. This module is highly configurable and supports all available sensor models, ranging from whiskbroom and pushbroom line scanners to frame cameras.

The input data for the orthorectification module is represented by sensor interior and exterior orientation, boresight calibration data and elevation data. The module not only outputs the position for every pixel in the image, but also produces the complete viewing geometry for every pixel, for seamless interfacing with MODTRAN (Berk et al. 1999), which is used for atmospheric correction of the imagery. Furthermore, it supports coordinate projection and datum transformations to any specified output coordinate system and datum. The output of the orthorectification module is a GIS formatted raster with 10 data layers: the $X, Y$ and $Z$ coordinates of every pixel, the solar zenith angle, the local illumination angle, the view zenith, solar azimuth, view azimuth, path length and the sky view factor. The algorithms involved in the orthorectification process are well-established and the corresponding original papers are indicated in the references section. The different aspects of the orthorectification are described in the following section.

The Orthorectification Module

In the following, we describe the input data of the orthorectification module, the core mechanism (ray tracing, time conversion, coordinate projection, spatial resampling), and the outputs produced by the module.
Kristin Vreys ${ }^{1}$, Marian-Daniel lordache ${ }^{2}$, Jan Biesemans ${ }^{3}$, Koen Meuleman

'Flemish Institute for Technological Research

(VITO NV), Belgium

e-mail: kristin.vreys@vito.be

${ }^{2}$ Flemish Institute for Technological Research

(VITO NV), Belgium

e-mail: marian-daniel.iordache@vito.be

${ }^{3}$ Flemish Institute for Technological Research

(VITO NV), Belgium

e-mail: jan.biesemans@vito.be

${ }^{4}$ Flemish Institute for Technological Research (VITO NV), Belgium

e-mail: koen.meuleman@vito.be

Received: 8 June 201

Accepted: 14 January 2016

\section{Sensor interior orientation}

There are two major types of line scanner: the pushbroom model and the whiskbroom model, each fully described by their specific parameters, as depicted in Figure 1.

Despite being designed as a pushbroom sensor, it turned out that APEX (APEX 2015) behaves as a whiskbroom sensor, implying that the field of view (FOV) fully defines the sensor geometry. The FOV was measured as part of the acceptance testing and found to be equal to 27.9842712347 degrees.

\section{Sensor exterior orientation}

Georeferencing of line scanner data requires accurate position $(X, Y, Z)$ and orientation ( $R=R o l l, P=P i t c h, Y=Y a w)$ data for every scanline in an image. For this purpose, real-time navigation data (logged on-board) is blended with GPS base station data (for differential correction) and installation parameters (GPS lever arms and IMU mounting angles) using the Applanix POSPAC MMS post-processing software (Hutton, Bourke \& Scherzinger 2007). This software outputs the so-called "Smoothed Best Estimated Trajectory (SBET)" file, providing the best possible position and orientation data for the time frame of the flight.

In addition to this, the boresight angles $(\Delta R, \Delta P, \Delta Y)$ are needed in the georeferencing process. These are the physical misalignment angles between the IMU and the sensor, as graphically presented in Figure 2 . They are determined during the boresight calibration (Mostafa 2001), which is a procedure intended to correct for angular misalignments between the sensor axis and the GPS/POS measurement system axis.

For APEX, a boresight calibration flight is performed over Ostend (Belgium) every time the instrument is newly built in the 


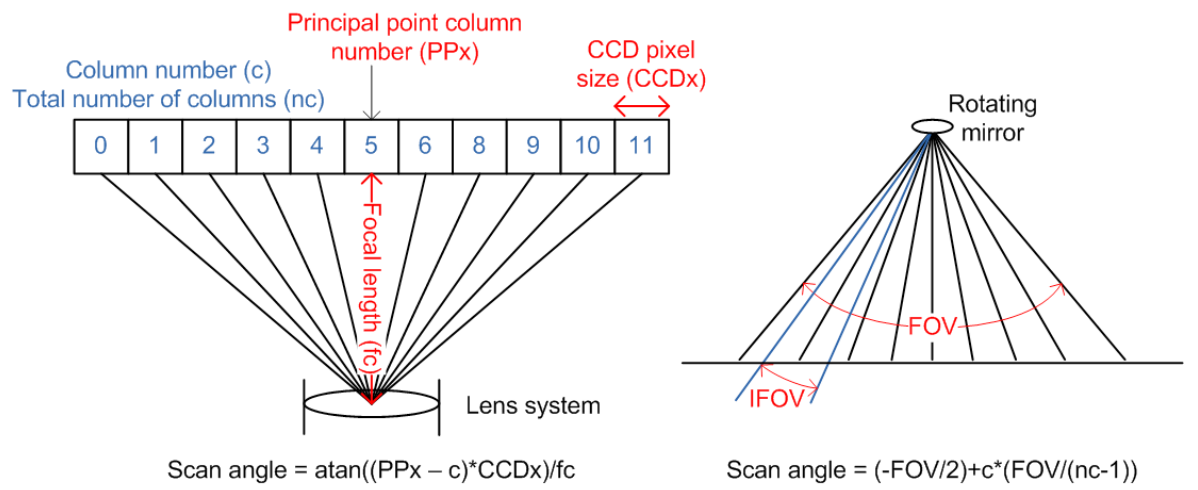

Figure 1. Interior orientation parameters (indicated in red) of the pushbroom (left) and whiskbroom sensor model

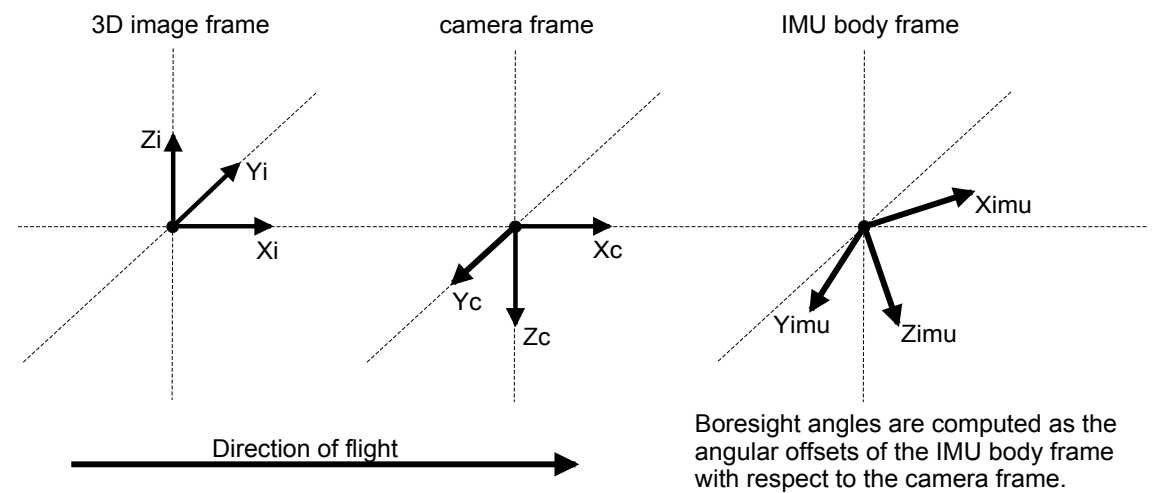

Figure 2. Image, camera and IMU reference frames (according to Abdullah, 2004)

aircraft. For this area, high-resolution orthophotos are available and reference ground control points (GCPs) are identified jointly with the corresponding pixels in the APEX imagery. The flight plan for the data acquisition mission over Ostend includes several overlapping flight lines from different directions and at various altitudes. Figure 3 shows a set of GCPs selected in the region. Note that preference is given to positions in non-homogeneous areas, where spatial transitions are visible (intersections, waterland neighbourhood), for easy identification in the APEX imagery.

A procedure based on Monte Carlo simulations is then employed to infer boresight angle corrections for the roll, pitch and yaw. Figure 4 plots the spatial deviation of APEX pixels (in metres) before (no boresight calibration performed, see top figure) and after boresight calibration (see bottom figure) for 117 GCPs. In this particular case, the calibration procedure reduced the mean deviation and its corresponding standard deviation from $17.76 \mathrm{~m}$ and $3.29 \mathrm{~m}$ to $1.56 \mathrm{~m}$ and $1.12 \mathrm{~m}$, respectively. It should be mentioned that the obtained deviation of $1.56 \mathrm{~m}$ is below the typical spatial resolution of APEX imagery, thus ensuring spatial displacements lower than one pixel. This value is in line with, or better than, displacements reported by other authors in the literature (Richter \& Schläpfer 2002, Holwartz, Müller \& Simon 2004).

Another visualization of the effect of the boresight calibration on the accuracy of the data geolocation is provided in Figure 5. In this figure, the geographic positions of the GCPs (red) are depicted jointly with the positions of the corresponding APEX pixels before and after boresight calibration (blue and green dots, respectively). From this figure, it can be observed that the roll, pitch and yaw corrections force the positions of the APEX pixels to approach the true ones.

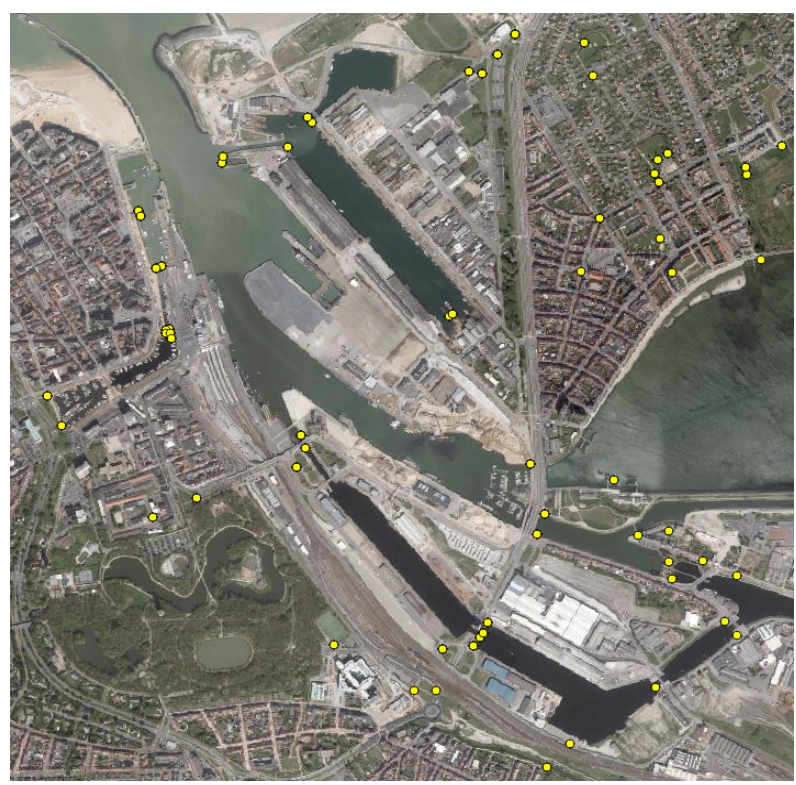

Figure 3. A set of GCPs (represented by yellow dots) in Ostend Source: <https://www.agiv.be/producten/orthofotomozaieken>

The inferred boresight calibration angles are then applied in the CDPC to all APEX imagery acquired before the sensor is unmounted from the aircraft. The periods in which APEX stays mounted on the flying platform depend on the aircraft availability. 

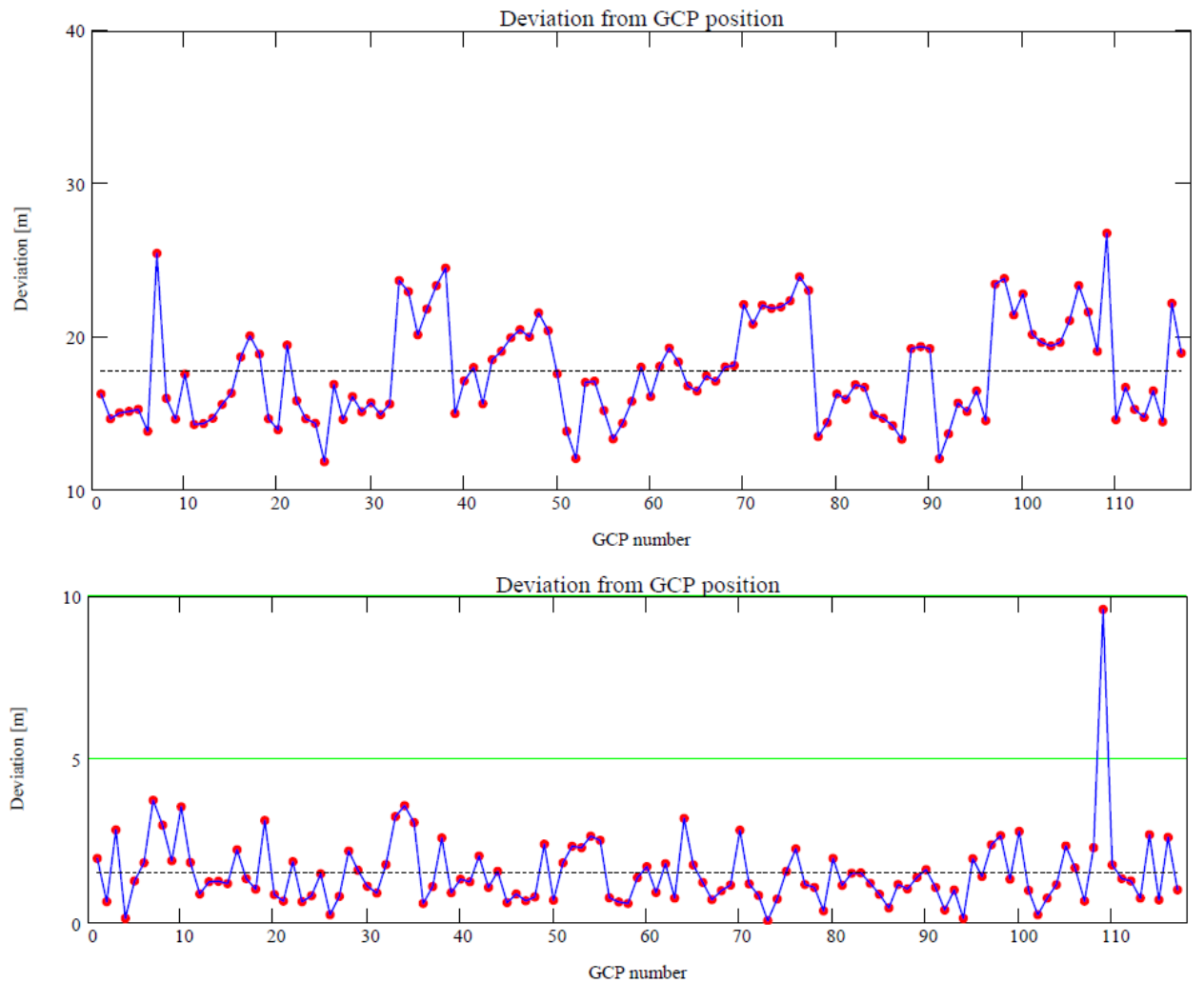

Figure 4. Spatial deviation (metres) of the APEX pixels from the corresponding GCPs before (top figure) and after (bottom figure) boresight calibration

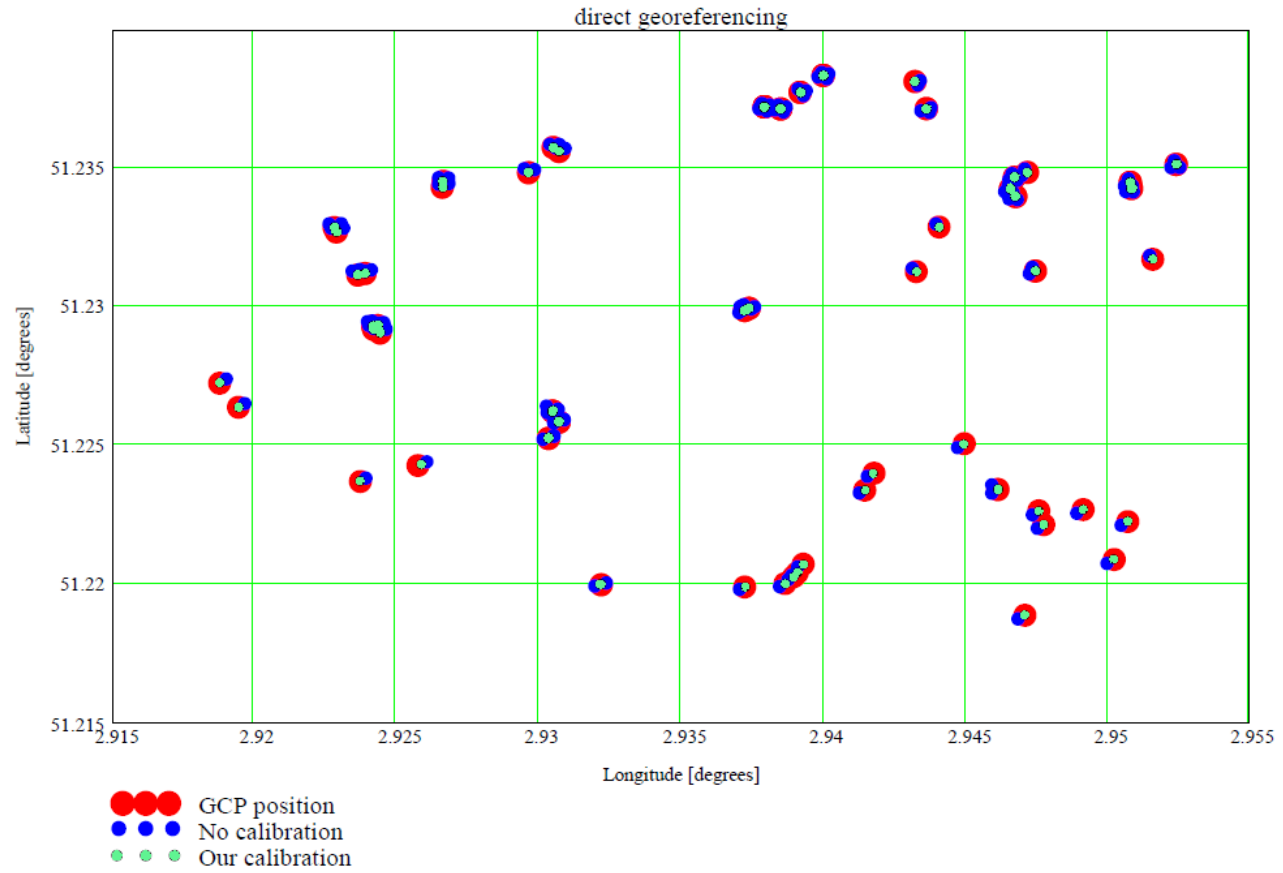

Figure 5. Geographic positions of the GCPs (red) and corresponding positions of APEX pixels for non-calibrated (blue) and calibrated (green) boresight angles 


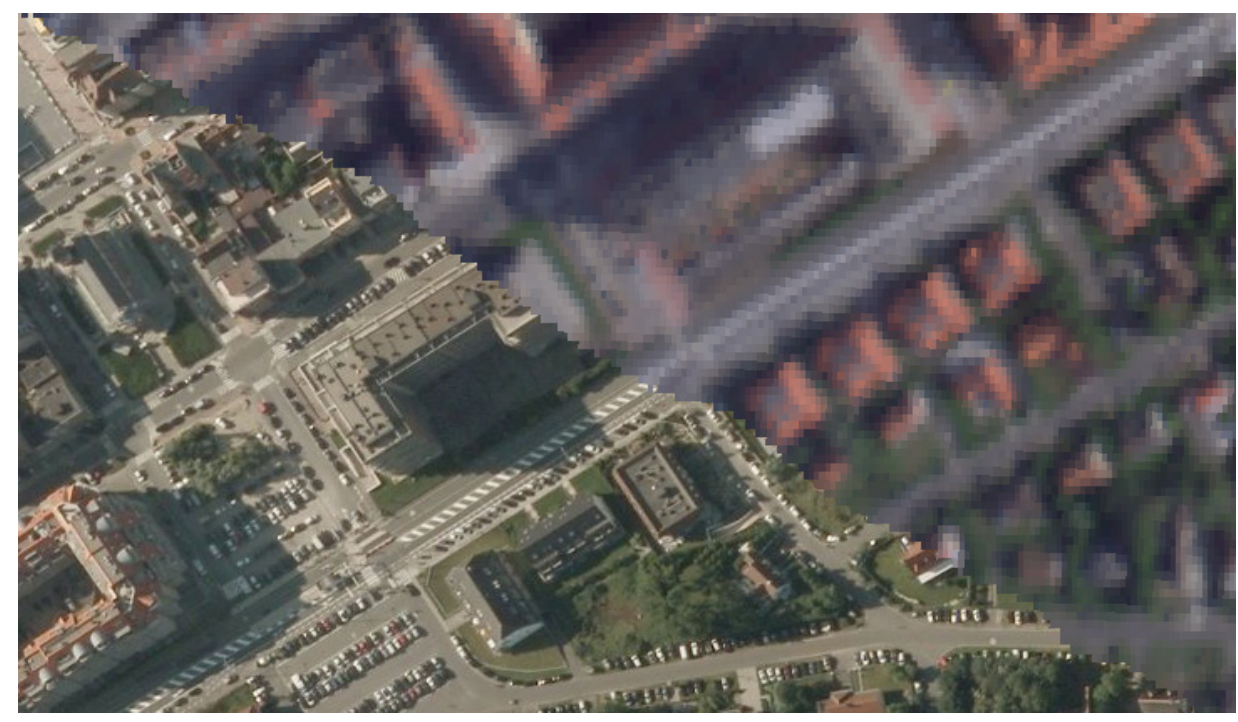

Figure 6. Example of an APEX image overlapped with the corresponding orthophoto in an urban area Source: <https://www.agiv.be/producten/orthofotomozaieken>

In one specific year, several time windows can be allocated to APEX flights. Thus, while all APEX imagery benefits from this procedure, the boresight calibration angles are updated from one year to another and even several times a year, if needed.

\section{Elevation data}

For the production of orthorectified APEX products, the following auxiliary elevation data layers are integrated in the Central Data Processing Center:

- The EGM968 geoid model (Lemoine et al. 1998). For direct georeferencing in the GPS coordinate system, the geoid is needed to retrieve the local geoid height which has to be added to the local DEM/DSM height.

- $\quad$ Above Flanders: the AGIV LIDAR DEM (DEM Flanders 2015) at a spatial resolution of $5 \mathrm{~m}$ and a vertical accuracy of $7 \mathrm{~cm}$ for areas covered with short grass or under pavement and $20 \mathrm{~cm}$ for areas under complex vegetation.

- Outside Flanders:

- A user-supplied (high resolution) LIDAR DTM/DSM, if available

- The SRTM DEM (the Shuttle Radar Topography Mission at a spatial resolution of $90 \mathrm{~m}$ ) (SRTM 2015) will be used as the fall-back mechanism if no detailed DEM is available

- The NOAA "GLOBE" global DEM (1 km spatial resolution) (NOAA 2015) is used as the fall-back mechanism to determine the mean elevation over the area covered by the image in case the SRTM DEM contains invalid or no data.

Time conversions

In the VITO orthorectification module, all time calculations (e.g. to link the APEX image data to the corresponding navigation data) are carried out in the GPS and UTC time systems. Whenever additional leap seconds are introduced, according to the bulletins published at LeapSeconds (2015), the module is updated. The most recent update of the UTC time was performed in June 2015 when GPS reached 17 seconds ahead of UTC time.

\section{Ray tracing}

The VITO module supports two manners of orthorectification: forward (direct georeferencing) or backward projection (indirect georeferencing). In forward projection, each pixel in the source image is projected onto the orthophoto. The corresponding pixels in the orthophoto are then determined via the intersection of a ray from the perspective centre of the source image through the image point with the three dimensional ground surface defined by the Digital Surface Model (DSM). In backward projection, each pixel in the orthophoto takes its pixel value from the source image using the collinearity condition and the object space coordinates $\mathrm{X}, \mathrm{Y}$, and $\mathrm{Z}$ of the corresponding DSM cell. Whether using forward or backward projection, full ray tracing is necessary to determine occluded areas (Abdullah 2004). The ray tracing is implemented according to the methodology described in reference documents (Möller \& Trumbore 1997; Amanatides \& Woo 1987).

Orthorectification of the APEX data is conducted via direct georeferencing in the GPS coordinate system (WGS84 datum and geographic coordinate system, i.e. latitude/longitude) and with the rotation angles given in roll, pitch and yaw (describing the rotation of the APEX sensor with respect to the ground).

\section{Solar position}

The orthorectification module outputs not only pixel positions but also the complete pixel viewing geometry. Therefore, detailed calculation of the solar position is required. For a complete description of the algorithm used to calculate the solar position, reference is made to Reda \& Andreas (2008). They based their implementation on the methodology presented in Meeus (1998) and their developed C source-code is publicly available (Reda \& Andreas 2008).

\section{Spatial resampling}

The spatial extent of the APEX pixels is dependent on the flight altitude (which determines the across-track resolution) and the sensor integration time, defined before the data acquisition mission according to user needs (influencing the along-track spatial resolution). The orthorectification module includes an automatic distance-weighted resampling and interpolation procedure to minimize spatial uncertainty (Galbraith, Theiler \& Bender 2003).

\section{Coordinate projection}

Coordinate and datum transformations are the very last step in the orthorectification. For the coordinate projection, the USGS 
GCTP package (USGS 2015) is used. This package is encapsulated in a $\mathrm{C}++$ interface developed at VITO and allowing for the addition of other projection systems not known by GCTP. For all datum transformations, the 7 parameter Helmert transformation is used (Helmet Transformation 2015).

\section{Example of a Geometrically Corrected APEX Image}

This section exemplifies the output of the VITO CDPC orthorectification module using remote sensing imagery acquired by APEX over an urban area. In Figure 6, the RGB representation of APEX imagery (in the upper part of the first diagonal of the image) is overlaid with a high-resolution orthophoto of the same area. A pixel-by-pixel analysis is not possible due to the relatively low spatial resolution of APEX in comparison to the high resolution reference image. However, note the excellent fit between the positioning of the APEX image and the background (e.g., the continuity of the streets and houses in the transition areas).

\section{Conclusions}

In this paper, the image orthorectification module implemented in the VITO CDPC was presented. The geometric correction of airborne imagery involves several procedures and components that have a significant impact on the final product. For APEX, an excellent absolute geolocation accuracy (sub-pixel to one pixel) is obtained after the orthorectification process when boresight calibration is performed. The examples shown in the paper confirm in a qualitative fashion the high accuracy of the APEX imagery from a geometrical point of view.

\section{References}

APEX hyperspectral sensor, 2015. Available from: <http://www. apex-esa.org/>. [28 January 2016].

Abdullah, QA 2004, 'Photogrammetric Platforms' in Manual of Photogrammetry, eds JC McGlone, EM Mikhail, J Bethel \& R Mullen, ASPRS, Bethesda, USA, pp. 677-730.

Amanatides, J \& Woo, A 1987, 'A fast voxel traversal algorithm for ray tracing', Eurographics '87, pp. 3-10.

Berk, A, Andersonm, GP, Bernstein, L, Acharya, P, Dothe, H, Matthew, M, Adler-Golden, S, Chetwynd, Jr, J, Richtsmeier, S, Brian Pukall, B, Allred, C, Jeong, L \& Hoke, M 1999, 'MODTRAN4 radiative transfer modeling for atmospheric correction', Proceedings SPIE 3756, Optical Spectroscopic Techniques and Instrumentation for Atmospheric and Space Research III, DOI:10.1117/12.366388 [20 October 1999].

Galbraith, AE, Theiler, JP \& Bender, SC 2003, 'Resampling methods for MTI coregistration product', Proceedings SPIE 5093 - Algorithms and Technologies for Multispectral, Hyperspectral and Ultraspectral Imagery IX, pp. 283-293.

Helmet transformation, 2015. Available from: <http://earth-info. nga.mil/GandG/coordsys/datums/helmert.html>. [28 January 2016].

High resolution LIDAR DEM over Flanders, 2015. Available from: <https://www.agiv.be/producten/digitaal-hoogtemodelvlaanderen>. [28 January 2016].

Holwartz, S, Müller, R \& Simon, C 2004, 'Determination and monitoring of boresight misalignment angles during the HyMap campaigns Hyeurope 2003 and Hyeurope 2004', Proceedings of 4th EARSeL Workshop on Imaging Spectroscopy. New quality in environmental studies, vol. 1, pp. 91-100.

Hutton, J, Bourke, T \& Scherzinger, B 2007, 'New developments of inertial navigation systems at Applanix', Proceedings of Photogrammetric Week, pp. 201-213.
LeapSeconds, 2015. Available from: <http://maia.usno.navy. mil/>. [28 January 2016].

Lemoine, FG, Kenyon, SC, Factor, JK, Trimmer, RG, Pavlis NK, Chinn, DS, Cox, CM, Klosko, SM, Luthcke, SB, Torrence, MH, Wang, YM, Williamson, RG, Pavlis, EC, Rapp, RH \& Olson, TR 1998, The development of joint NASA GFSC and NIMA geopotential model EGM96, NASA Goddard Space Flight Center, Maryland, USA.

Meeus, J 1998, Astronomical Algorithms, Second Edition, Willmann-Bell, Inc., Richmond, Virginia, USA.

Mostafa, MMR 2001, 'Boresight calibration of integrated inertial/camera systems', Proceedings of the International Symposium on Kinematic Systems in Geodesy, Geomatics and Navigation - KIS 2001, Banff, Canada, pp. 440-445.

Möller, T \& Trumbore, B 1997, 'Fast, minimum storage raytriangle intersection', Journal of Graphics Tools, vol. 2, issue 1, pp. 21-28.

NOAA 2015, Global DEM. Available from: <http://www.ngdc. noaa.gov/mgg/topo/globe.html>. [28 January 2016].

Reda, I \& Andreas, A 2008, 'Solar position algorithm for solar radiation applications', National Renewable Energy Laboratory, report NREL/TP-560-34302. Available from: <http://www.nrel.gov/midc/spa/>. [4 December 2015].

Richter, R \& Schläpfer, D 2002, 'Geo-atmospheric processing of airborne imaging spectrometry data. Part 2: Atmospheric/ topographic correction', International Journal of Remote Sensing, vol. 23, no. 13 , pp. 2631-2649.

Shuttle Radar Topography Mission (SRTM), 2015. Available from: <http://srtm.usgs.gov/>. [28 January 2016].

USGS 2015, GCTP package. Available from: <http://edcftp. cr.usgs.gov/pub//software/gctpc/>. [28 January 2016]. 